\title{
Fundus albipunctatus associated with cone dystrophy
}

\author{
Yozo Miyake, Noriyasu Shiroyama, Shintaro Sugita, Masayuki Horiguchi, Katsuya Yagasaki
}

\begin{abstract}
We describe five unrelated patients in whom the typical signs of fundus albipunctatus were accompanied by colour vision defects, bull's eye or similar macular lesions, and severely diminished full-field cone electroretinograms indicating widespread damage to cones outside the macula. All patients had noticed night blindness from childhood. Signs of retinitis punctata albescens, a disease of similar appearance but with characteristics resembling retinitis pigmentosa, were absent. We cannot be sure whether these patients represent a process of fundus albipunctatus or a distinct disease entity or a casual combination of fundus albipunctatus and cone dystrophy.
\end{abstract}

Fundus albipunctatus is a stationary disorder characterised by myriads of yellow-white dots in the fundus and an abnormally slow rate of dark adaptation. ${ }^{1-6}$ The electroretinograms (ERGs) require a much longer dark adaptation time than normal to reach maximum amplitude.

Some reports have described cases of macular degeneration associated with fundus albipunctatus. ${ }^{17-10}$ In the past 10 years we have had five patients in whom apparent fundus albipunctatus was associated with macular degeneration. In all the patients the rod function showed characteristic properties of fundus albipunctatus, but full-field cone ERGs showed significant reduction.

\section{Case reports}

The five unrelated patients had noticed night blindness from childhood. The anterior segment, lens, and vitreous were normal in all patients, and none showed nystagmus or strabismus. The

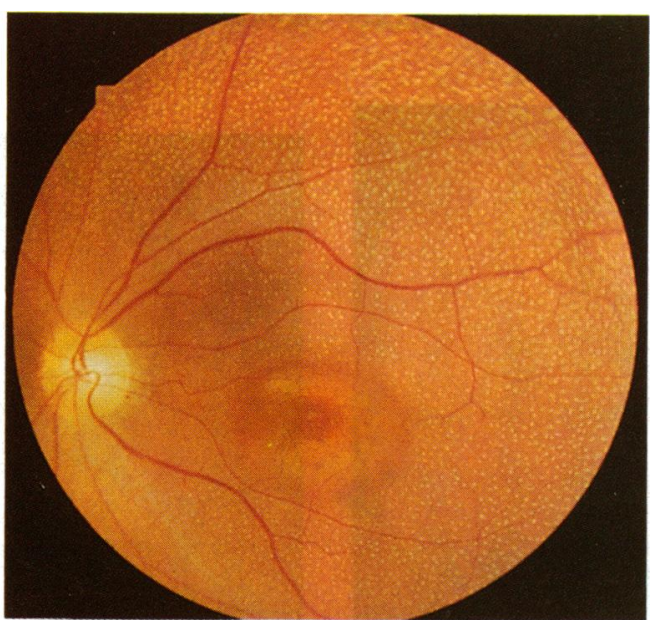

photograph $(A)$ and fluorescein angiography $(B)$ of the left eye in case 1 .

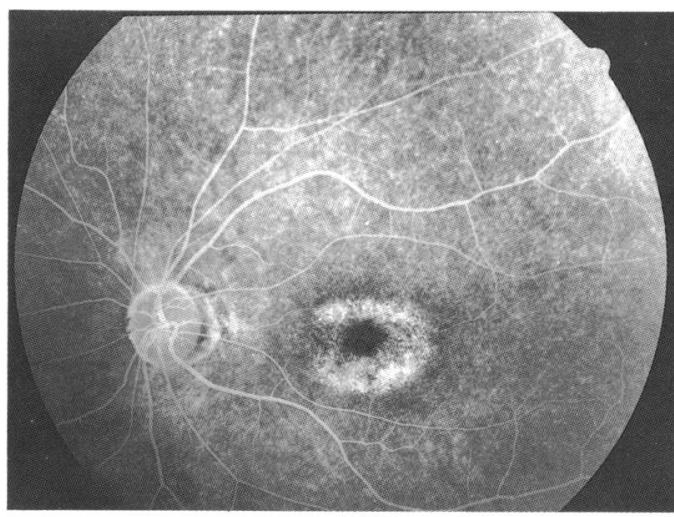

Fig $1 B$

results of general physical examinations and routine laboratory tests were also normal.

CASE 1

A 49-year-old man with night blindness had noticed reduced vision over the previous 6 years; his corrected visual acuity was 0.6 in each eye. No one with night blindness or severely low vision was found in his pedigree. His healthy parents were first cousins.

Discrete, dull white, uniform, dot-like lesions, seen over much of the fundi, were of great density in the midperiphery of both eyes (Fig 1A). The maculae each showed a bull's eye lesion. No attenuation of retinal vessels, optic atrophy, or pigmentary clumping was seen. Fluorescein angiography revealed irregular, spotty hyperfluorescence in the midperiphery, which did not correspond to the flecks; the macula showed ring-shaped hyperfluorescence (Fig 1B). Visual fields were tested with the Goldmann perimeter using 3 ioptres. The peripheral visual fields were normal, but central sensitivity was slightly decreased in both eyes. Colour vision tests revealed severe blue-yellow and red-green defects. Dark adaptation was tested by using the Goldmann-Weeker adaptometer in the right eye. A white test target of $11^{\circ}$ was presented at $15^{\circ}$ of the upper retina. After 30 minutes the visual threshold was approximately $5 \log$ units higher than normal. After 3 hours it remained 0.5 to $1.0 \log$ unit higher than normal.

Full-field ERGs of the right eye (Fig 2) were made by the standard recording technique previously described." The mean (with 2 SD) amplitudes in the normal subjects after 30 minutes of dark adaptation were 325 (140) $\mu \mathrm{V}$ (scotopic (rod) b wave), $59(21) \mu \mathrm{V}$ (photopic (cone) b wave), and $38(19 \cdot 1) \mu \mathrm{V}(30 \mathrm{~Hz}$ flicker responses). After 30 minutes of dark adaptation 
Figure 2 Full-field electroretinograms of a normal subject and cases 1 to 5 . In each case the upper tracings indicate the responses recorded after 30 minutes of dark adaptation, the lower tracings indicate the responses recorded after prolonged dark adaptation (see text for the details). The arrows indicate the stimulus onset.

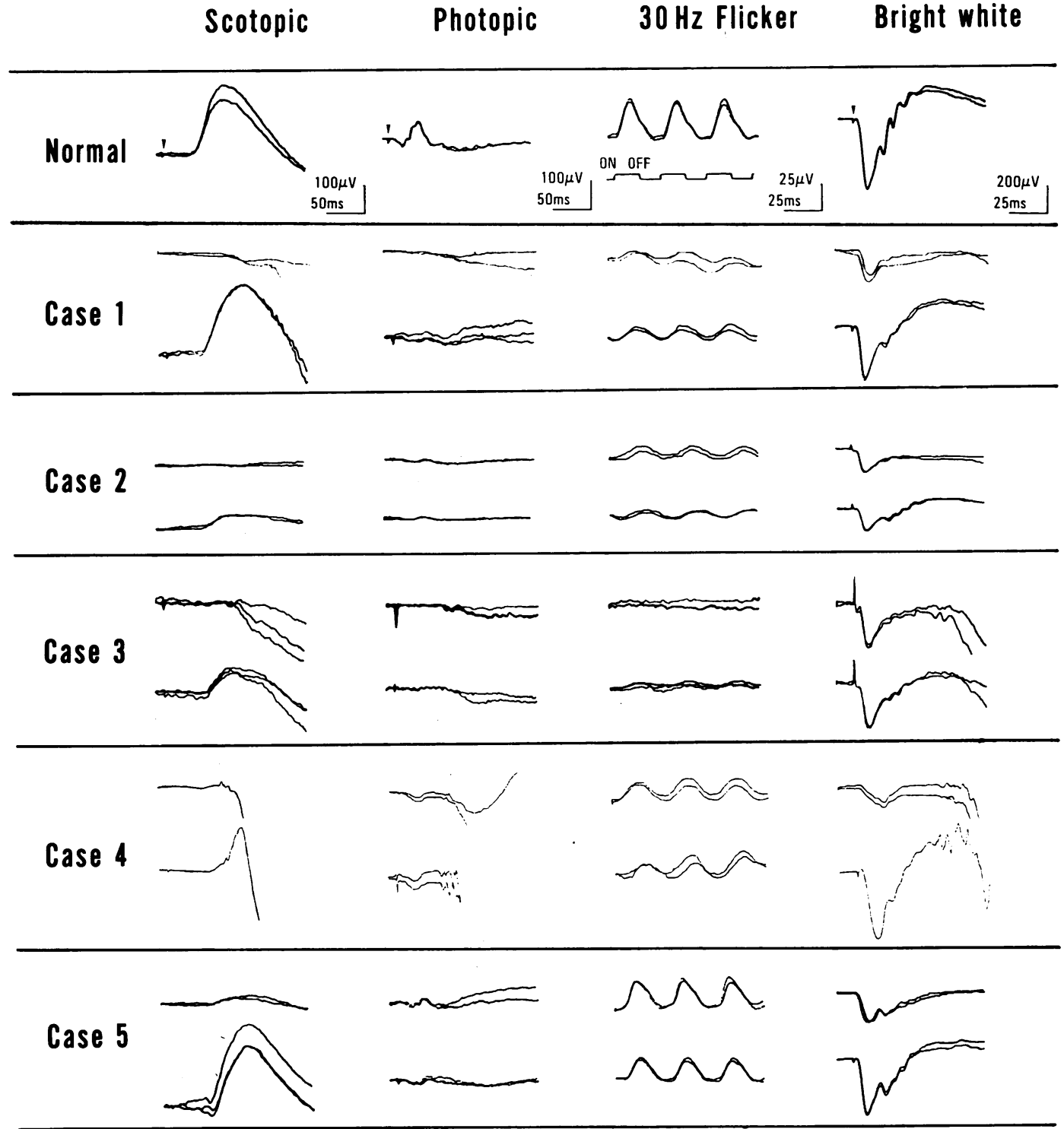

(rod) or photopic (cone) ERG was recordable. The amplitudes of $30 \mathrm{~Hz}$ flicker responses were $9.6 \mu \mathrm{V}$ with the delayed implicit time. A single bright-flash (white bright) ERG showed severely decreased $b$ wave and moderately decreased a wave. After 3 hours of dark adaptation (Fig 2, lower tracings in case 1), rod b wave was $291 \mu \mathrm{V}$ in amplitude with normal implicit time. The cone $b$ wave was $16.6 \mu \mathrm{V}$ in amplitude with delayed implicit time. The $30 \mathrm{~Hz}$ flicker

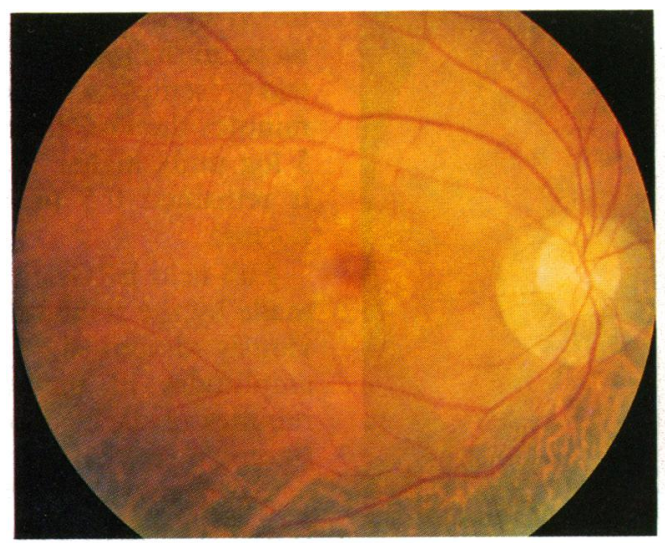

Fig $3 A$ responses showed little change in comparison with those recorded after 30 minutes of dark adaptation. A single bright-flash ERG showed normally shaped a waves and $b$ waves.

An electro-oculogram (EOG) was recorded as described previously." The light peak versus dark trough ratio was 1.42 (right eye) and 1.37 (left eye). A statistical analysis of normal subjects indicates that a ratio smaller than 1.80 is abnormal.

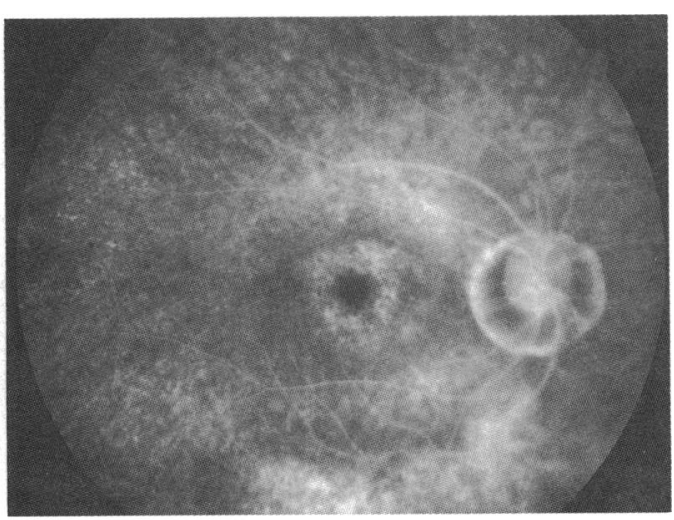




\section{CASE 2}

A 49-year-old man with night blindness first noticed blurred vision and metamorphopsia in both eyes in 1987. In 1989 his corrected visual acuity was 1.2 (right eye) and 1.0 (left eye), and in 1990 it was 0.6 (right eye) and 1.0 (left eye). No patient with night blindness was found in his pedigree, and his parents were not consanguineous.

The fundus and fluorescein angiography findings (Figs $3 \mathrm{~A}, \mathrm{~B}$ ) were similar to those of case 1 . However, a localised, symmetrical chorioretinal atrophy was noted in the nasal inferior fundus of both eyes. Suggestive attenuation of retinal vessels was observed, and the optic disc was normal in both eyes. The peripheral visual fields were normal except for a depression in the area corresponding to the chorioretinal atrophy; the central visual fields each had an annular scotoma. Colour vision tests revealed a severe blue-yellow defect and moderate red-green defect. The visual threshold was 3.8 log units higher than normal after 30 minutes of dark adaptation and was $2 \cdot 5$ log units higher than normal after 1 hour of dark adaptation.

In full-field ERGs recorded in the right eye after 30 minutes of dark adaptation (Fig 2, upper tracings in case 2 ), rod b wave was unrecordable, while cone b wave $(15 \mu \mathrm{V})$ and $30 \mathrm{~Hz}$ flicker responses $(9.6 \mu \mathrm{V})$ were extremely reduced with delayed implicit time. A single bright-flash ERG showed severely decreased $b$ and moderately decreased a waves. After 1.5 hours of dark adaptation (Fig 2, lower tracings in case 2) the rod $b$ wave was recorded with an amplitude of $67 \mu \mathrm{V}$ with normal peak time. Single brightflash electroretinography recorded increased $b$ wave amplitude. The cone and $30 \mathrm{~Hz}$ flicker responses were not significantly different from those recorded after 30 minutes of dark adaptation.

The EOG ratios were 1.75 (right eye) and 1.64 (left eye).

\section{CASE 3}

This patient with night blindness was first examined by us in 1981 at the age of 40 . There was no notable family history except that his parents were first cousins. The fundus appeared to be typical of fundus albipunctatus, and the maculae were normal ophthalmoscopically. In 1984 we found some pigmentary clumpings in the nasal inferior area of the right eye which developed into local chorioretinal atrophy during a further 6-year follow-up.

In 1989 we noted ophthalmoscopic changes, suggestive of early bull's eye maculopathy, in the maculae of both eyes (Fig 4). Fluorescein angiography also showed suggestive, ring-shaped hyperfluorescence.

In 1990 the visual acuity was 1.2 in each eye. The peripheral visual fields showed depression only in the area corresponding to the chorioretinal atrophy in the right eye. The central field showed slightly decreased central sensitivity in both eyes. Colour vision tests revealed a moderate blue-yellow defect and mild red-green defect. The visual threshold during dark adaptation was 4.0 log units higher than normal after 30 minutes of dark adaptation, but reached a normal level after 3 hours of dark adaptation.

Full-field electroretinography (Fig 2, upper tracings in case 3 ) did not record rod, cone, or 30 $\mathrm{Hz}$ flicker responses after 30 minutes of dark adaptation. A single bright-flash ERG showed mildly and severely reduced $a$ and $b$ waves, respectively. After 3 hours of dark adaptation (Fig 2, lower tracing in case 3) a rod b wave of amplitude of $116 \mu \mathrm{V}$ with normal peak time was recorded. Cone b wave and $30 \mathrm{~Hz}$ flicker responses were extremely small, with the amplitudes of $14 \mu \mathrm{V}$ and $12 \mu \mathrm{V}$, respectively, with the delayed implicit times. Single bright-flash electroretinography showed increased $b$ wave amplitude.

The EOG ratios were $2 \cdot 52$ (right eye) and $2 \cdot 23$ (left eye).

\section{CASE 4}

In 1984 a 34-year-old man with night blindness visited our clinic because he noticed reduced vision in his left eye; visual acuity was 1.0 (right eye) and 0.4 (left eye). In 1988 the visual acuity was $1 \cdot 0$ (right eye) and $0 \cdot 1$ (left eye). His younger brother complained of night blindness, but was not examined. Their parents were not consanguineous.

His fundus (Fig 5A) contained multiple yellowwhite dots mainly in the midperiphery; the maculae showed well circumscribed chorioretinal atrophy of 1.5 disc diameters, which spared the foveola in the right eye (Fig 5B). The optic disc was normal, and the retinal vessels were not attentuated. No pigment clumps were seen in the fundus. The visual fields were essentially normal except for a central scotoma of $7^{\circ}$ to $9^{\circ}$ detected in each eye. Colour vision tests indicated severe red-green defect and moderate blue-yellow defect. The dark adaptation curve was $4.7 \mathrm{log}$ units above normal threshold after 30 minutes and $2 \log$ units above normal after 2 hours of dark adaptation.

Full-field ERGs after 30 minutes of dark adaptation (Fig 2, upper tracing in case 4) showed no rod response and reduced cone $b$ wave and $30 \mathrm{~Hz}$ flicker responses $(25 \mu \mathrm{V}$ and $15.4 \mu \mathrm{V}$, respectively). The implicit times of cone $b$ wave and $30 \mathrm{~Hz}$ flicker responses were delayed. Single bright-flash electroretinography recorded reduced a and b waves. After 2 hours of dark adaptation (Fig 2, lower tracings in case 4 ) rod b wave was recorded (amplitude, 
Figure 5 Fundus photograph $(A)$ and fluorescein angiography $(B)$ of the left eye in case 4.

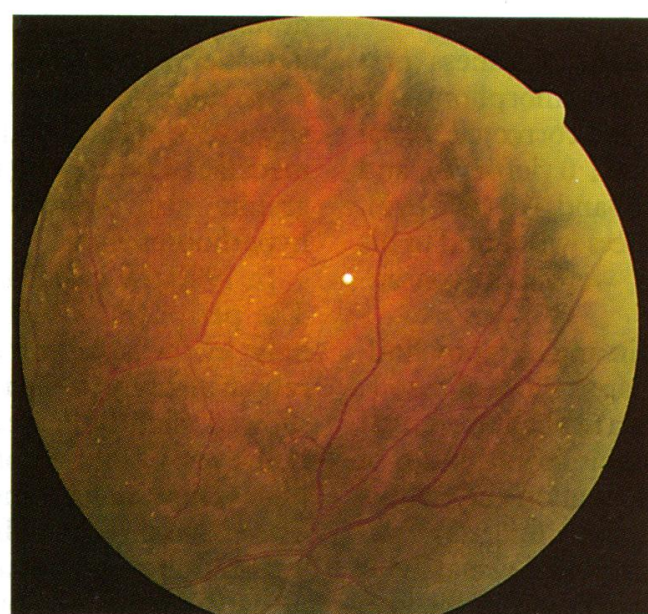

Fig 5 A

$192 \mu \mathrm{V}$ ) with normal implicit time. The cone $\mathrm{b}$ wave and $30 \mathrm{~Hz}$ flicker responses showed no significant change after 2 hours' dark adaptation; single bright-flash ERG showed an increase in amplitude of a waves and $b$ waves.

The EOG ratios were normal: $1 \cdot 86$ (right eye) and $2 \cdot 15$ (left eye).

CASE 5

A 37-year-old woman with night blindness noticed reduced vision about 10 years ago. In 1987 her corrected visual acuity was 0.8 (right eye) and $0 \cdot 4$ (left eye). Her pedigree contained no other patient with night blindness or low visual acuity, and her parents were not consanguineous.

Fundus examination (Fig 6A) revealed multiple white dots in the midperiphery and non-specific degeneration of the macula. Fluorescein angiography showed irregular hyperfluorescence in the macula (Fig 6B). The peripheral visual field was full, and the central field showed slightly decreased sensitivity. Colour vision tests indicated severe red-green and moderate blue-yellow defects. The dark adaptation curve was $5.5 \mathrm{log}$ units above normal threshold after 30 minutes of dark adaptation but was normal after 3 hours of dark adaptation In full-field ERGs recorded after 30 minutes of dark adaptation (Fig 2, upper tracings in case 5), the amplitudes were $33 \mu \mathrm{V}$ (rod b wave), $30 \mu \mathrm{V}$ (cone b wave) and $27 \mu \mathrm{V}(30 \mathrm{~Hz}$ flicker responses). Their implicit times were normal. Single bright-flash electroretinography revealed

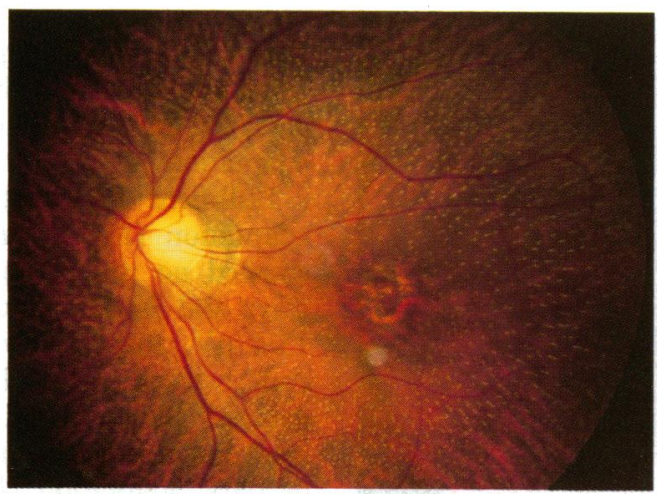

Fig 6A

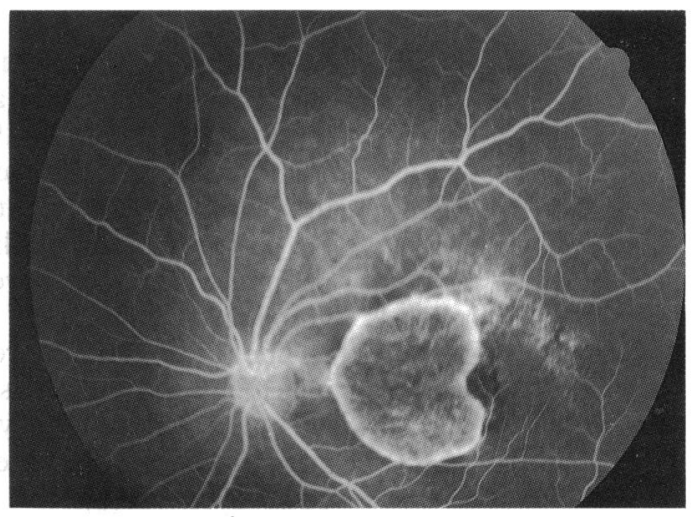

Fig $5 B$

moderately reduced a wave and severely reduced b wave. After 3 hours of dark adaptation the rod ERG and a single bright-flash responses were normal. Cone ERG and $30 \mathrm{~Hz}$ flicker responses, however, remained unchanged.

\section{Discussion}

The five patients described here had ocular signs of both fundus albipunctatus and cone dystrophy. Bull's eye or similar macular lesions were present, and full-field cone electroretinograms demonstrated that damage to cones was not limited to the macula, but was widespread as in cone dystrophy. All patients had noticed night blindness from childhood. They started to notice decreased visual acuity from the second or the third decade, and we therefore believe that the cone impairment is progressive. All these patients had severe colour vision defects, even though some of them had relatively good visual acuity.

Fundus albipunctatus with macular degeneration has been reported by other authors. ${ }^{7-10}$ Detailed ocular examinations of two patients ${ }^{9-10}$ yielded findings similar to ours. The fundi of these patients displayed multiple yellowish white dots, and the maculae showed bull's eye maculopathy. Recovery of rod function was abnormally delayed and cone electroretinograms showed severe impairment.

Retinitis punctata albescens is a hereditary dystrophy which produces white dots in the fundus and the clinical and electrophysiological characteristics of retinitis pigmentosa. ${ }^{12-14}$ The punctate lesions are similar to those in fundus albipunctatus, but the fundus also shows diffuse atrophy of the retinal and pigment epithelium,

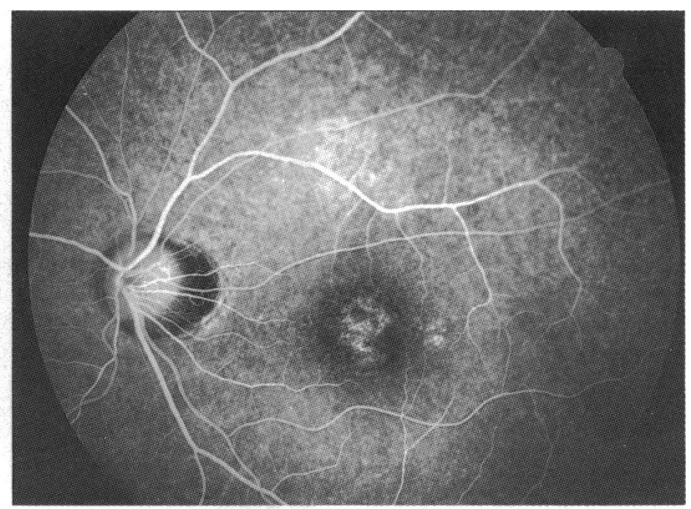

Fig $6 B$ 
arterial narrowing, disc pallor, and often some black pigment spicules. The symptoms include permanent night blindness, peripheral field loss, and sometimes a loss of central vision. Some authors consider that these disorders form a continuum, but convincing evidence indicates they are separate entities. Using fundus reflectometry to study two brothers with fundus albipunctatus, Carr and associates ${ }^{15}$ noted that the slow dark adaptation that characterised the disorder clinically corresponded to an abnormally slow rate of visual pigment regeneration. Thus our concept of fundus albipunctatus and retinitis punctata albescens is that the former is a stationary disorder of visual pigment regeneration, while the latter is a progressive dystrophy that permanently damages the photoreceptors and pigment epithelium.

Our patients did not have retinitis punctata albescens, because they had abnormally delayed recovery of rod function, no attenuation of retinal vessels (only case 2 showed suggestive attenuation of retinal vessels), no disc pallor, and a normal or only slightly constricted peripheral visual field. Moreover, it should be noted that the rod $\mathrm{b}$ wave after prolonged dark adaptation showed normal implicit time in all patients. It seems more likely that fundus albipunctatus was associated with cone dystrophy. Two of our patients (cases 2 and 3) showed some pigment spicules, localised in the midperipheral retina. Cone dystrophy may also show such changes during the course of progression.' In the advanced stage of cone dystrophy rods may also be affected, retinal vessels become attenuated, and pigment clumps and diffuse atrophy of the retinal pigment epithelium become apparent.
Once these characteristics have developed, the differential diagnosis from retinitis punctata albescens may not be easy.

At present we cannot establish unequivocally whether these patients represent a process of fundus albipunctatus or an independent clinical entity or simply the combined manifestations of two different diseases.

Presented at the Annual Meeting of the Association for Research in Vision and Ophthalmology, Sarasota, Florida, USA, 30 April 1991.

1 Krill AE. Hereditary retinal and choroidal diseases. Hagerstown: Harper and Row, 1977; 2: 739-824.

2 Carr RE. Congenital stationary night blindness. Trans $\mathrm{Am}$ Ophthalmol Soc 1974; 72: 448-87.

3 Marmor MF. Dystrophies of the retinal pigment epithelium. In: Zinn KM, Marmor MF, eds. The retinal pigmen epithelium

4 Francescheti A, Francois J, Babel J. Chorioretinal heredodegeneration. Springfield: Thomas, 1974; 222-50.

5 Vannas S, Setala $M$. On atypical night blindness. Aannas S, Setala M. On atypical
Acta Ophthalmol $(K b h)$ 1958; 36: 849-59.

6 Miyake Y, Asano T, Sakai T, Watanabe I. ERG and EOG in retinitis punctata albescens. Acta Soc Ophthalmol fpn 1972; 76: $247-56$.

7 Gass JDM. Stereoscopic atlas of macular diseases. Diagnosis and treatment. 2nd ed. St Louis: Mosby, 1977: 184-5.

8 Scouras J, Chevaleraud J, Papastratigakis B. Macular involve ment in retinitis albescens. F Fr Ophtalmol 1979; 2: 613-7.

9 Weleber RG. Retinitis pigmentosa and allied disorders. In Ryan SJ, Ogden TE, eds. Retina. St Louis: Mosby, 1989; 1 346-8.

10 Sato S, Iijima $\mathrm{H}$. A case of fundus albipunctatus associated with cone dysfunction. Rinsho Ganka 1987; 41: 1307-10.

11 Miyake Y, Yagasaki K, Horiguchi M, Kawase Y, Kanda T. Congenital stationary night blindness with negative electroretinogram. A new classification. Arch Ophthalmol 1986; 104: 1013-20.

12 Lauber H. Die sogenaunte retinitis punctata albescens. Klin Monatsbl Augenheilkd 1910; 48: 133-48.

13 Tamai A, Setogawa T, Kandori F. Electroretinographic studies on retinitis punctata albescens. Am $\mathcal{F}$ Ophthalmo 1966; 62: 125-31.

14 Ellis D, Heckenlively J. Retinitis punctata albescens. Fundus appearance and functional abnormalities. Retina 1983; 3: 27-31.

15 Carr RE, Ripps H, Siegel IM. Visual pigment kinetics and adaptation in fundus albipunctatus. Doc Ophthalmol Proc Ser 1974; 4: 193-204. 